\title{
A motion aftereffect for long-range stroboscopic apparent motion
}

\author{
MICHAEL W. VON GRÜNAU \\ Queen's University, Kingston, Ontario, Canada
}

\begin{abstract}
The existence of a directional motion aftereffect (MAE) for long-range (LR) stroboscopic apparent motion (SAM) was examined with the use of a directionally ambiguous test stimulus. The spatial and temporal parameters were such that the LR, rather than the short-range, mechanism was likely to be implicated. MAEs were found for SAM, which were in the same direction, but somewhat weaker than those for a comparable stimulus in real motion. The MAEs for SAM were present only when good apparent motion was perceived, and could be shown also when only the unstimulated area between the two stroboscopic flashes was tested. The LR mechanism was further implicated, since the MAEs were also obtained under dichoptic adaptation conditions. It is concluded that the LR-motion mechanism does show a usual MAE under proper testing conditions.
\end{abstract}

In the study of perception, much use has been made of illusions, that is, instances in which our perceptual experience does obviously not correspond to the physical stimulus situation. It was the Gestalt school which initiated this emphasis, but the tradition is still very much alive today. In the perception of motion, two illusions have been studied very extensively: the motion aftereffect (MAE) and stroboscopic apparent motion (SAM). In the former, typically a stationary stimulus appears to move in a direction opposite to the one presented during an adaptation phase (Wohlgemuth, 1911). In the latter, the presentation of typically two stationary stimuli with appropriate spatial and temporal separations leads to the perception of continuous smooth motion (Wertheimer, 1912).

Although the MAE is a very strong and reliable effect in the case of real motion, that is, in the situation in which a stimulus is actually drifting across the retina, the effect is not as clearly established in the case of SAM. There have been several reports in the literature that have described MAEs for various stroboscopic motion stimuli (e.g., Anstis \& Mather, 1985; Banks \& Kane, 1972; Beck \& Stevens, 1972; Bennett \& Westheimer, 1985; Mather, Cavanagh, \& Anstis, 1985), but the displacements used to produce SAM, for which a MAE was present, were usually very small. For larger displacements, MAEs either could not be observed (Anstis \& Mather, 1985; Mather et al., 1985) or were very weak and lasted only 2 or $3 \mathrm{sec}$ (Anstis \& Moulden, 1970). This difference has been used by Anstis (1980) as one of the defining factors that can

This research was supported by NSERC Grant U 0057 and ARC equipment grants (Queen's University) to M.W.v.G. I would like to thank Andrea Riendeau and Andrea Korda for their help with the experiments, and Patrick Cavanagh for comments on the manuscript.

Part of this research was presented at the 7th European Conference of Visual Perception in Cambridge, U.K., 1984.

The author's present address is: Departement de psychologie, Université de Montréal, C.P. 6128, succursale A, Montréal, Québec, Canada H3C $3 \mathrm{~J} 7$. discriminate between the short-range (SR) and long-range (LR) motion mechanisms. According to a suggestion by Braddick (1974), the SR mechanism codes real motion as well as SAM with small displacements and short interstimulus intervals (ISIs). The LR mechanism, on the other hand, is concerned with SAM when displacements are larger and ISIs are longer.

Recently, it has been shown that these two motion mechanisms (SR and LR) are not always independent, but can interact with each other under certain conditions. In a direct test, Green (1983) pitted real and LR apparent motion against each other and found that real motion could totally inhibit or strongly facilitate SAM, depending on whether the two motions were in the opposite or same direction. Green and von Grünau (1983) extended this finding to the MAE. Adaptation to a drifting stimulus could enhance or inhibit LR SAM, depending on the direction of the adapting drift (see also Gregory \& Harris, 1984).

Thus, the SR and LR motion mechanisms do not seem incompatible. The present experiments, therefore, attempted to explore the possibility that LR apparent motion could also produce appreciable and robust MAEs if tested appropriately, that is, in such a way that the presentation of the test stimulus would not immediately destroy the SAM percept. The reported results indicate that the LR motion mechanism can have a MAE, which is similar in direction to, but weaker than, the MAE produced by the SR motion mechanism.

\section{GENERAL METHOD}

The general idea of the present experiments was to adapt the visual system either to a drifting grating or a grating patch in stroboscopic apparent LR motion or to a stationary control stimulus. The common test for the effects of these three adaptation conditions was a stationary grating patch that suddenly reversed its spatial phase. The perceived direction of this phase shift is theoretically ambiguous; that is, without directional adaptation or any other directional 
bias, it should be seen to shift in either direction for about $50 \%$ of the trials.

\section{Apparatus}

The stimulus display was generated on the face of a Tektronix 602 CRT display monitor with the help of an Innisfree image synthesizer which was under the control of an Apple II+ microcomputer. The dimensions of this display are indicated in Figure 1. A $12.2^{\circ} \times 5^{\circ}$ area of the screen was masked out and divided into three windows that were separated by thin paper strips. The three windows were under individual control, so that different stimuli could be presented to each window. A fixation circle was continuously present beneath the display. The stimuli consisted of vertical sinusoidal gratings with a contrast of $8 \%$, an average luminance of $.8 \mathrm{fL}$, and a spatial frequency of .5 or $1.0 \mathrm{cpd}$ at the observation distance of $57 \mathrm{~cm}$.

\section{Procedure}

For each experimental session, the observers were dark-adapted to the general illumination of the lab, which was in the mesopic range. They steadied their heads on a chin/foreheadrest and fixated the fixation circle. When they were ready, they initiated the first adapatation period by pressing a button. The adaptation stimulus was presented for $30 \mathrm{sec}$, after which the test stimulus appeared. This consisted of a stationary grating, which was presented for $216 \mathrm{msec}$, after which it switched spatial phase abruptly by $180^{\circ}$, remained for another $216 \mathrm{msec}$, and then was replaced by a homogeneous surface of .8-fL mean luminance. At this point, the observers had to indicate, by pressing the appropriate button, in which direction the test stimulus had shifted. The response was recorded by the computer, and the observers had to initiate the next adaptation period. This sequence was repeated until 10 responses had been made. Only one adaptation condition was presented per day, and the order was counterbalanced, so that each condition was presented four times.

Before the experimental sessions, the observers' optimal ISI values were determined by presentation of the SAM display (as described in detail in Experiment 1) for a range of 10 ISI values in randomized order, so that each ISI value appeared 10 times. This was done for left as well as right motion. The observers indicated whether they perceived smooth continuous motion after each presentation. The responses were recorded and tallied by the computer.

\section{Observers}

The observers consisted of the author and a number of undergraduate students who were naive as to the purpose of these experiments but were trained extensively in the tasks of these experiments and given much practice with the SAM display, so that they felt comfortable in making the required judgments. All had normal or corrected-to-normal spatial vision.

\section{EXPERIMENT 1}

\section{Method}

The particular stimulus display for Experiment 1 is shown in Figure 1. For the STROBE adaptation condition, a grating patch appeared in one of the two outside windows for $48 \mathrm{msec}$ and was followed, after the optimal ISI, by the same patch in the other outside window, also for $48 \mathrm{msec}$. Between the end of the second flash and the reappearance of the first flash there was a long interval (ITI) of $500 \mathrm{msec}$, so that unidirectional motion was achieved. An example for rightward motion is illustrated in Figure 1. The center window, which always remained at an average luminance of $.8 \mathrm{fL}$, was $4.2^{\circ}$ wide. Thus, apparent motion had to occur across this distance, which should be large enough to stimulate the $\mathbf{L R}$ mechanism. For the DRIFT adaptation condition, the grating drifted across the whole field with a velocity of $16 \mathrm{deg} / \mathrm{sec}$ for a spatial frequency of $.5 \mathrm{cpd}$.

For the STATIONARY control adaptation condition, a stationary grating appeared in the whole field for the same amount of time as was used in the other two conditions. The temporal sequence for all three conditions is shown in Figure 2. All adaptation stimuli were presented intermittently. The time for each presentation was

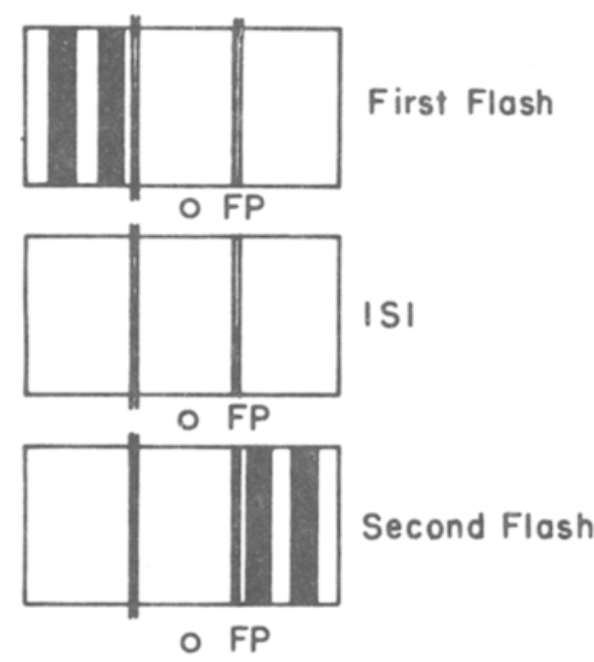

STROBE (right motion)

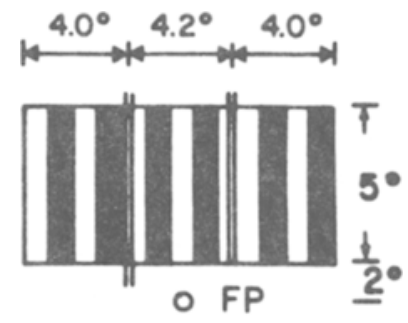

DRIFT (right motion)

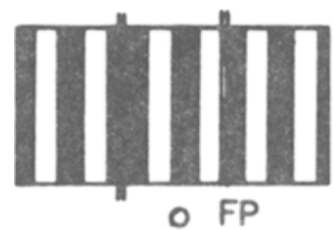

STATIONARY and TEST

Figure 1. Stimulus display for Experiments 1 and 2 with dimensions in degrees of vianal angle. (Left) A stroboscopic motion sequence (STROBE) for motion to the right; (right top) display for real motion (DRIFT); (right bottom) display for the stationary control and test stimuli. The narrow parallel vertical lines dividing the display into three parts indicate strips of paper. FP = fowtion circle. The gratings were actually sinusoidal and of low contrast. 
A DAPEATIOH TES T

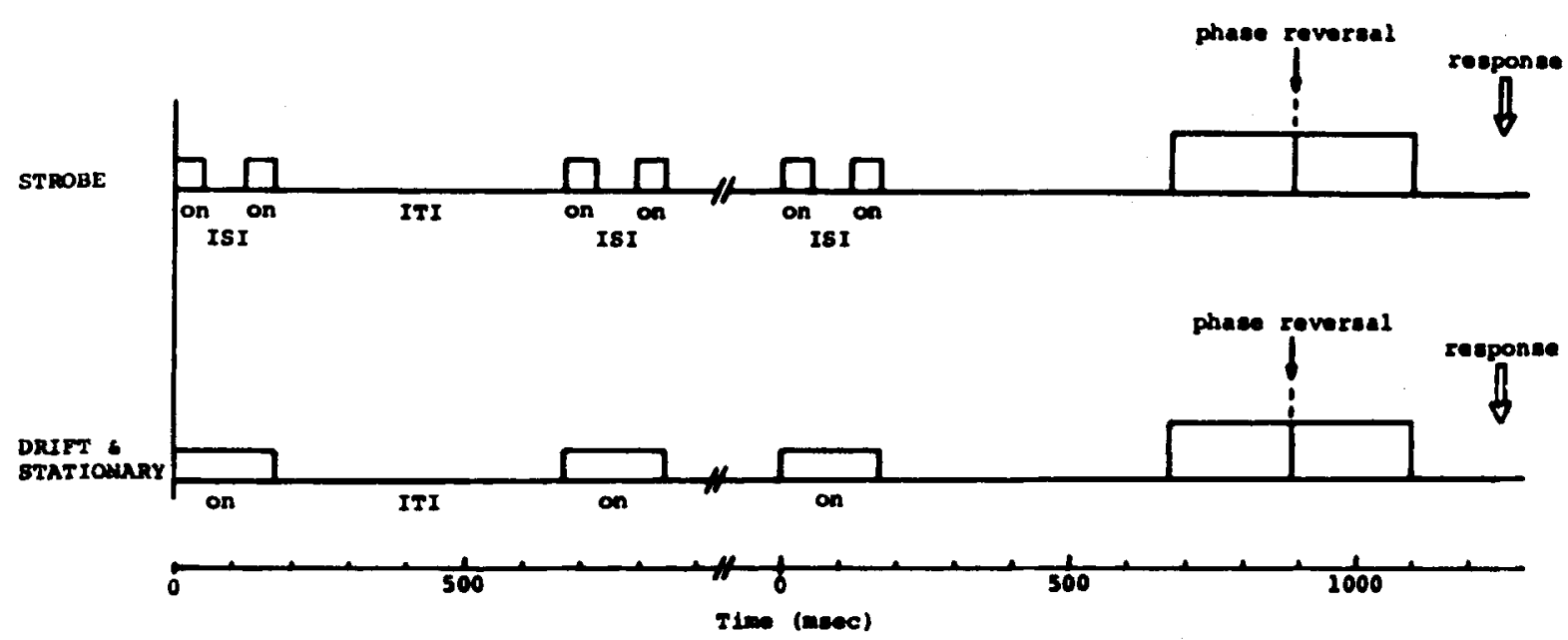

Figure 2. Temporal arrangement for adaptation and test periods for STROBE (top) and DRIFT and STATIONARY (bottom) conditions. "on" indicates the presentation time for the stimuli.

determined by the optimal ISI and amounted to ISI $+96 \mathrm{msec}$. The next presentation followed after an ITI of $500 \mathrm{msec}$. This sequence was repeated unit $30 \mathrm{sec}$ were up and was then followed by the test.

\section{Results and Discussion}

Figure 3 presents the results used to determine the optimal ISI for one observer. The percentage of reports of "smooth motion" is graphed as a function of ISI. The relationship is characterized by the typical bell-shaped curve. The optimal ISI is indicated by the left arrow. Similarly, all observers had their own individual optimal ISI values.

The results for Experiment 1 are presented in Figure 4 as the average for the 3 observers, since all performed very similarly. The responses could be either "left" or "right," and the percentage of "left" responses is graphed for the two experimental adaptation conditions and for the control adaptation condition. For DRIFT adaptation (left panel), motion to the left resulted in a sharp reduction and motion to the right in a large increase in the percentage of "left" responses, so that adaptation was almost complete in either case $\left(P\left\{\chi^{2}>33.9\right\} \ll .01\right)$. This result shows the presence of strong MAEs for this kind of adaptation, the direction of which depended on the direction of the adaptation. For STROBE adaptation (right panel), the outcome was very similar $\left(P\left\{\chi^{2}>\right.\right.$ $13.26\}<.01)$, but the effects were not as strong $\left(P\left\{\chi^{2}\right.\right.$ $>28.36\} \ll .01)$. Yet, again, MAEs were present, and this biased the perceived direction of motion in exactly the same way. Experiment 1, then, provides evidence that adaptation to LR apparent motion can lead to a directional $\mathrm{AE}$, which is similar to, but not as strong as, the $\mathrm{AE}$ for real motion. Such a comparison might be justified in the present case, since both real and apparent motion were presented in comparable ways during adaptation.

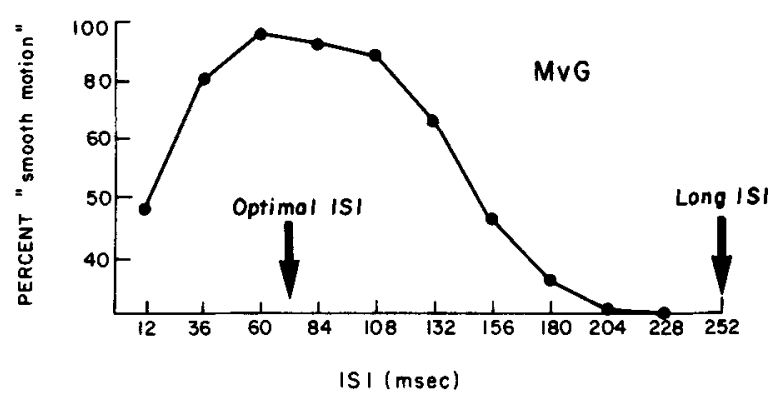

Figure 3. Likelihood of seeing "smooth continuous motion" as a function of ISI for 1 observer. The left arrow indicates the time chosen for this observer for optimal motion perception (optimal ISI $=72 \mathrm{msec}$ ); the right arrow points to the time used for the perception of succession (long ISI $=252$ msec). Data points are based on 30 observations each.

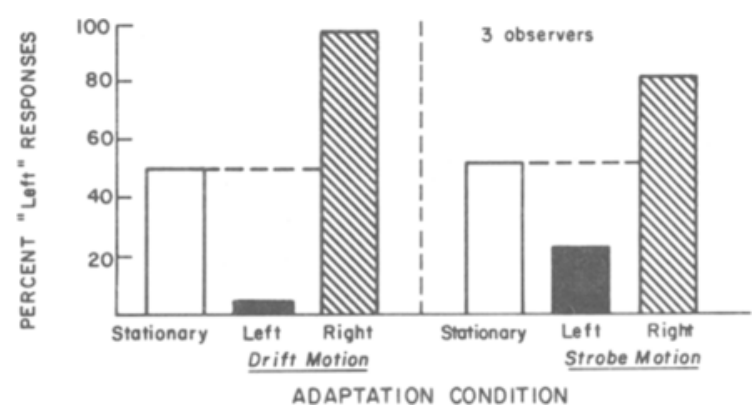

Figure 4. The percentage of "left" responses to the apparent direction of the test-grating phase shift as a function of adaptation condition for Experiment 1. Responses for stationary control and left (L) and right (R) motion are given for DRIFT and STROBE motion. Data are the averages for 3 observers. Each column is based on 120 observations. 


\section{EXPERIMENT 2}

On the one hand, Experiment 2 was a replication of Experiment 1 with different observers. On the other hand, it also provided important control conditions. In Experiment 1 , the conditions for apparent motion were chosen to optimize the perception of motion by using the optimal ISI. Here, new conditions were added, including very long ISI values ( $252 \mathrm{msec}$ ), which lay far beyond the range for which motion was seen. The conditions were such that the grating patches were clearly seen in succession with no motion. This situation is indicated in Figure 3 by the right arrow for long ISI.

\section{Method}

In this experiment, the stationary control condition was presented before and after each run of the motion adaptation conditions. Thus, each direction of adaptation, as well as the two ISI values and the two types of motion, had their individual controls. In all other respects, Experiment 2 was like Experiment 1. Three observers participated, 2 of whom were naive as to the purpose of this experiment and had participated in the previous experiment.
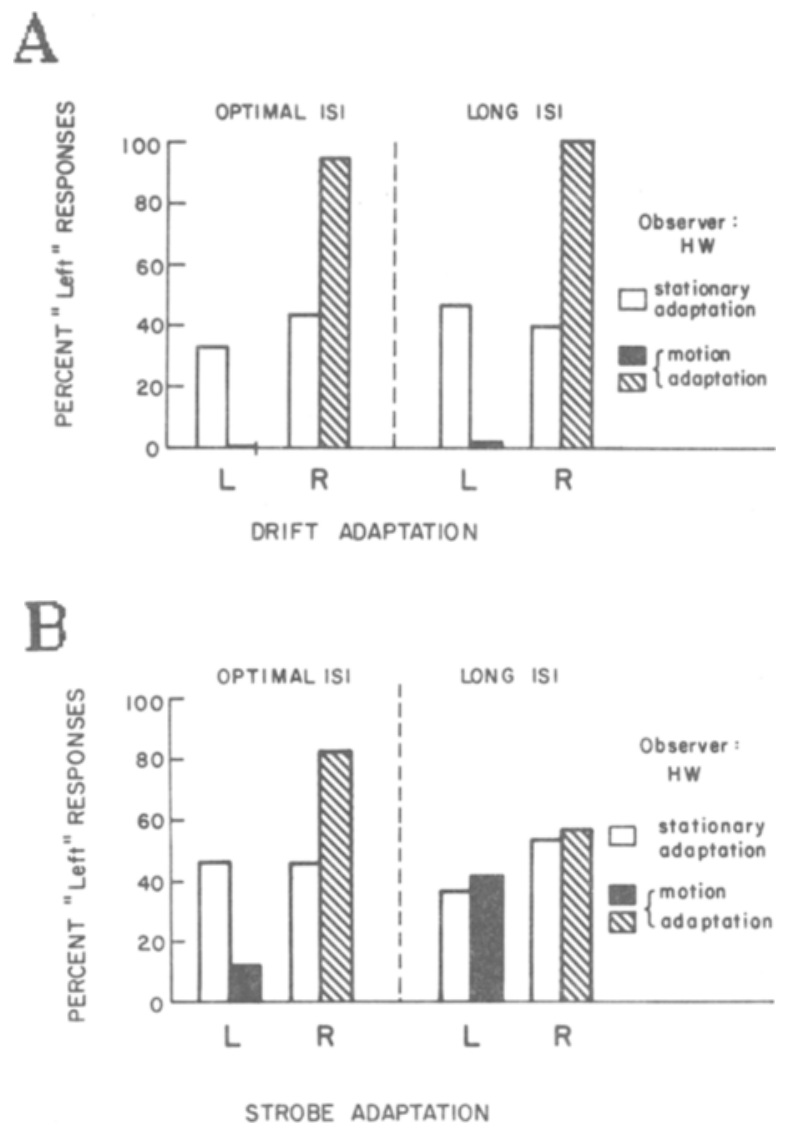

Figure 5. Percentage of "left" responses for DRIFT adaptation (A) and STROBE adaptation (B) for Experiment 2. Data are from 1 typical observer. Each motion direction ( $L$ and $R$ ) is compared with its stationary control. The left side of the figure is for optimal motion (optimal ISI), and the right side is for succession (long ISI). Data are based on $\mathbf{4 0}$ responses for each column.

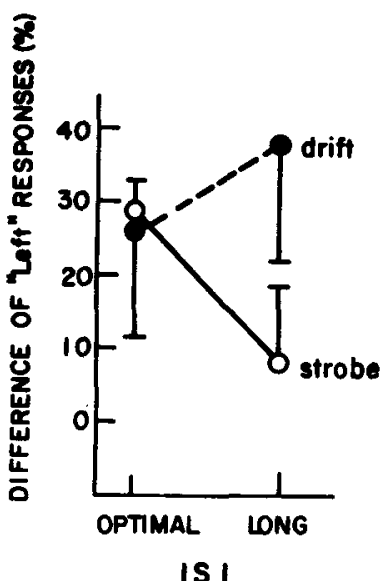

Figure 6. Summary of Experiment 2. To combine the data for all 3 observers, the percent difference of "left" responses in each condition to the appropriate control condition is graphed as a function of ISI (optimal $=$ best motion, long $=$ no perceived motion). For DRIFT and STROBE motion, the results for the two directions are also averaged. Each data point is, therefore, based on 240 observations. Vertical bars indicate 1 standard deviation.

\section{Results and Discussion}

Since the results for all 3 observers were essentially the same, the results presented in Figure 5 are for only 1 of the new observers. The top panel is for DRIFT adaptation. On the left, results are graphed for the optimal ISI value, which replicated those of Experiment 1. Adaptation to a leftward drift reduced the number of "left" responses, whereas adaptation to a rightward drift increased them $\left(P\left\{\chi^{2}>38.75\right\} \ll .01\right)$. The results for the long ISI on the right were very similar, but somewhat stronger $\left(P\left\{\chi^{2}>53.3\right\} \ll .01\right)$. This was to be expected, since, for DRIFT motion, the longer ISI just meant longer adaptation periods, and, thus, the stronger MAE (Sekuler \& Pantle, 1967).

The corresponding results for the STROBE adaptation are displayed in the lower panel. Again, the results for the optimal ISI on the left replicate those of Experiment 1 $\left(P\left\{\chi^{2}>21.46\right\} \ll .01\right)$. They provide evidence for the existence of a MAE for LR apparent motion. That this aftereffect depended on the actual perception of motion in the stroboscopic stimulus is shown by the results for the long ISI on the right. No aftereffect appeared for either direction of adaptation $\left(P\left\{\chi^{2}>.39\right\}>.5\right)$.

These results are summarized in Figure 6 as the averages for all 3 observers. Leftward and rightward adaptations were combined by using the difference between the "left" responses to the experimental and corresponding control condition and plotting the mean. For optimal ISI, both motion adaptations showed MAEs that were about equally strong and not significantly different. For long ISI, this was not the case; the MAE increased slightly for DRIFT adaptation, but practically disappeared for STROBE adaptation. Thus, Experiment 2 confirmed the existence of a MAE for LR apparent motion and demonstrated that this was tied to the perception of motion. 

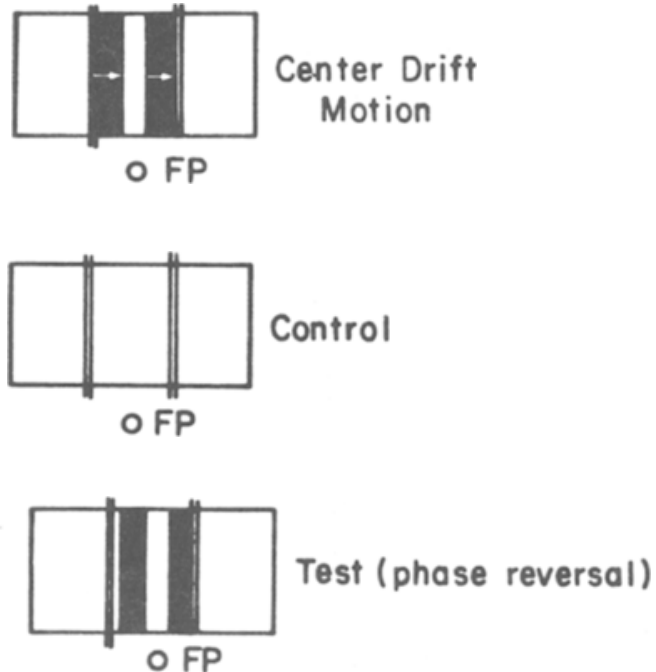

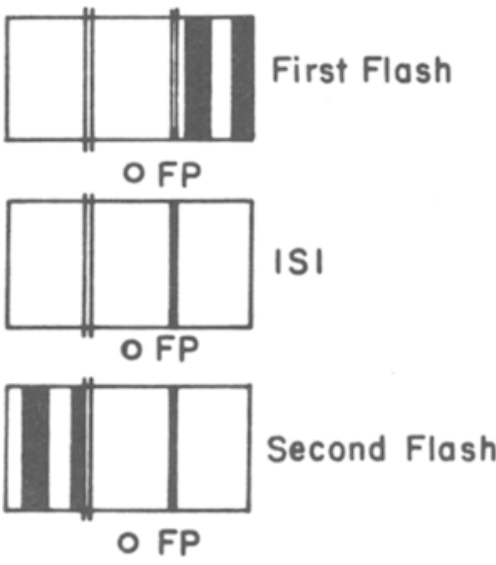

Strobe Motion

Figure 7. Stimulus display for Experiment 3. On the left are the conditions for drifting motion, control, and test. On the right is an apparent motion sequence for motion to the left. Actual gratings were sinusoidal and of low contrast. $\mathbf{F P}=$ fxation circle. Dimensions are as in Figure 1.

\section{EXPERIMENT 3}

In the first two experiments, the test stimulus was large and overlapped with the areas that were stimulated by the grating patches in the STROBE adaptation condition. In this experiment, only the central part of the display was examined.

\section{Method}

The display for Experiment 3 is presented in Figure 7. For the DRIFT motion adaptation (here called center drift motion), the drifting grating was presented only in the central window of the display (top left). The other two windows were at average luminance. For the control condition, all three windows were at mean luminance, so that adaptation was to a homogeneous field. Otherwise, the adaptation parameters were the same as before (center left). The STROBE motion display remained the same as in the previous two experiments. An example of leftward motion is indicated on the right of Figure 7. The test stimulus was confined to the center window (bottom left). Otherwise, the timing and procedure of adaptation and test presentations were the same as before. The experiment was run for two spatial frequencies of the gratings, 0.5 and $1.0 \mathrm{cpd}$.

Two observers participated in this experiment; 1 of them was naive as to the purpose of the study, but was well practiced in the tasks. The other observer was the author.

\section{Results and Discussion}

The results are presented as the average for the $2 \mathrm{ob}$ servers in Figure 8. Again, they are graphed as the percentage of "left" responses to the perceived direction of the test grating shift for the various adaptation conditions. The results for the control condition are indicated by a stippled line. For the center drift motion condition, the obtained MAEs were very strong for both directions and spatial frequencies $\left(P\left\{\chi^{2}>33.2\right\} \ll .01\right)$. For the STROBE motion condition, MAEs were also present
$\left(P\left\{\chi^{2}>9.97\right\}<.01\right)$, but they were not as strong $\left(P\left\{\chi^{2}\right.\right.$ $>32.98\} \ll .01)$. It is, however, clear that a directiondependent bias was introduced by the adaptation to SAM also in this situation, where test and adaptation stimuli did not overlap in physical space. In other words, this experiment demonstrates that evidence for a MAE can be found, even if only the physically unstimulated area between the two grating patches is tested. That is, the perceived direction of an apparently moving stimulus can bias directional mechanisms in the visual system.

This statement is true to the extent that eye movements were small and kept to a minimum. Even though no monitoring of eye movements was undertaken in these experiments, the following steps were taken to minimize the effects of eye movements: A fixation circle was continuously present. All observers were well trained in the experimental tasks, which included long periods of fixation. The three stimulus windows were separated by $.2^{\circ}$-wide strips. Any overlap must, therefore, have been very small.

\section{EXPERIMENT 4}

The stimulus display for the present experiments was chosen such that the LR motion mechanism was likely to have been stimulated (Braddick, 1974). Since it was imperative for the present investigation that we deal with LR motion, another defining characteristic was examined in this experiment. According to earlier studies (Anstis, 1980; Braddick, 1974; but see Shadlen \& Carney, 1985, for a cyclopean SR motion), SR motion does not survive dichoptic presentation of the inducing stimuli, whereas LR motion occurs even under those conditions. This may be expected if it is assumed that the LR mechanism is more central, at a stage where information from both eyes is available, but that this is not the case for the more 


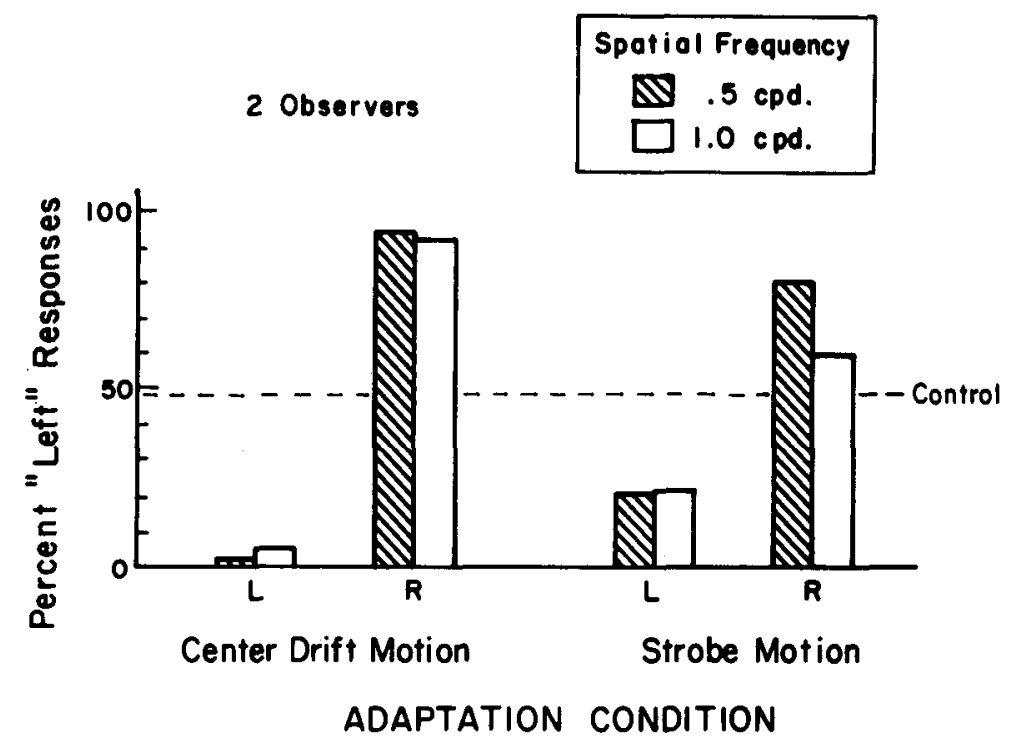

Figure 8. Percentage of "left" responses as a function of adaptation condition (Experiment 3). Results for two spatial frequencies are given and averaged over the 2 observers for left (L) and right (R) motion for center DRIFT (left) and STROBE (right) motion. The stippled line is for the results of the homogeneous control adaptation condition. Each column is based on $\mathbf{8 0}$ observations.

peripheral SR mechanism. In Experiment 4, therefore, the two grating patches were presented dichoptically, and the presence of a MAE was tested in ways similar to those used previously.

\section{Method}

The stimulus display was like the one used in Experiment 3 (see Figure 7). Only STROBE motion adaptation was used, with grating patches that had a spatial frequency of $0.5 \mathrm{cpd}$. The control adaptation stimulus was again a homogeneous field with a luminance of $0.42 \mathrm{fL}$, which was also the mean luminance of the grating displays. Again, the MAE was tested only in the central window. Dichoptic presentation was achieved by using polarizing filters in front of the two outside windows. The two polarization planes were at right angles, and the observers wore glasses with the same kind of polarizing filters, which were oriented in such a way that the left grating patch could be seen only with the left eye and the right patch only with the right eye. The test patch in the center could be seen by both eyes.

There were five adaptation conditions: the control condition (with the timing determined by optimal ISI), leftward and rightward STROBE motion with optimal ISI, and leftward and rightward STROBE motion with long ISI. The optimal ISI was determined individually for each observer before the experiment, and the long ISI was set at $252 \mathrm{msec}$. For each of the adaptation conditions, 40 responses were collected and grouped in runs of 10 . The $5 \times 4$ runs were counterbalanced and run on separate days.

Three observers participated. One was the author. The other 2 were naive as to the purpose of the experiment, and 1 of them had not participated in any of the previous experiments. All received extensive practice with the required tasks.

\section{Results and Discussion}

The results for Experiment 4 are displayed in Figure 9 for all 3 observers, since the size of the effect varied considerably among them. All 3 observers, however, were consistent in terms of the direction of the effects, and these were, therefore, analyzed together. STROBE motion with optimal ISI produced directional MAEs for all observers $\left(P\left\{\chi^{2}>4.16\right\}<.05\right)$. The condition with long ISI, in which no motion was perceived, again gave no MAEs, just as in the previous experiment $\left(P\left\{\chi^{2}>.04\right\}>.8\right)$. Observer A.R. (panel C) showed an overall bias for leftward motion superimposed over the otherwise similar results. The great differences between observers may be attributable to the difficulty of the perceptual task.

The outcome of this experiment provides further evidence that the LR motion mechanism was stimulated under the conditions of the present experiments. It follows that the obtained MAEs are also attributable to the LR motion mechanism.

\section{DISCUSSION}

The present series of experiments attempted to show the existence of a directional MAE for SAM under conditions that were conducive for the operation of the LR motion mechanism. The results that were consistently obtained demonstrate that the perceived direction of a directionally ambiguous test stimulus could be biased by prolonged inspection of a LR apparent motion stimulus. This bias was in the same direction as the one found for prolonged inspection of a stimulus in real motion, that is, the perceived direction was always opposite to the direction of the adaptation stimulus. The size of the aftereffect, however, was usually not as large as for real motion.

Accepting the existence of an aftereffect in the present situation still leaves unanswered the important question about which motion mechanism had actually been adapted. 

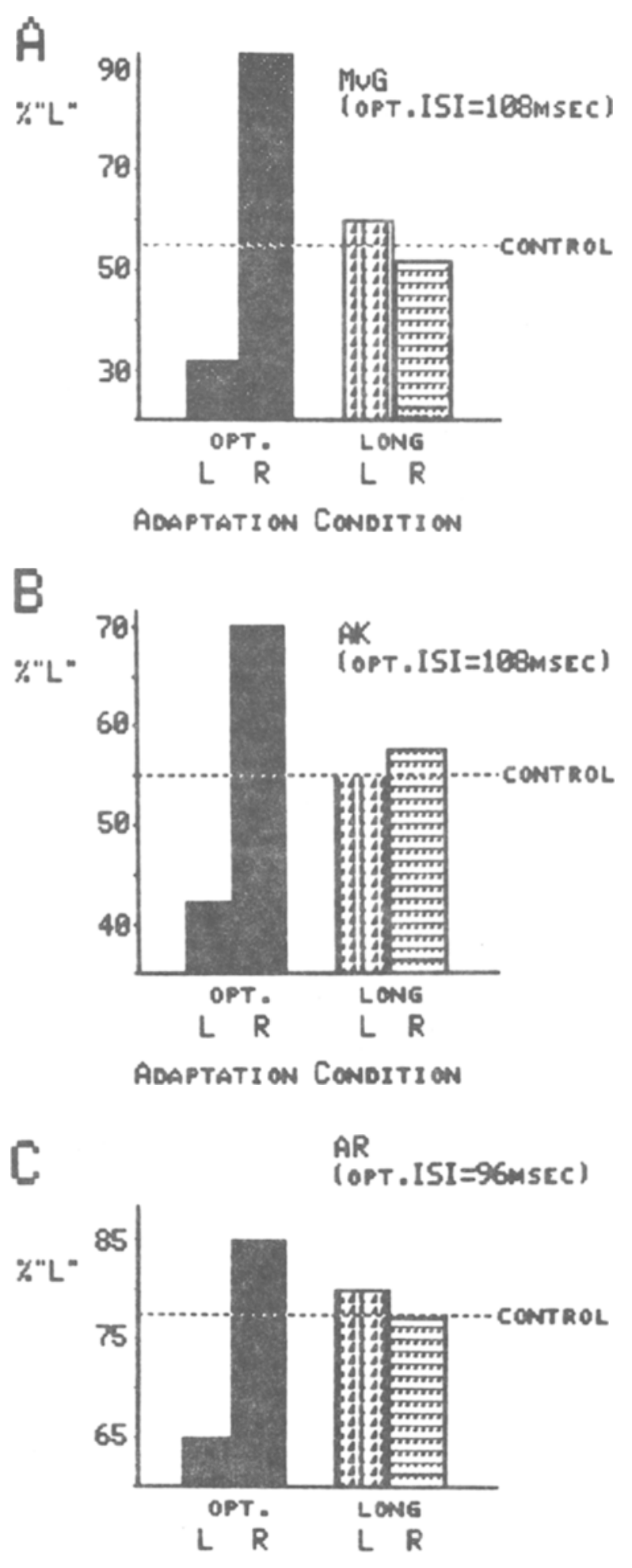

ADaptation CONDITION

Figure 9. Percentage of "left" responses as a function of adaptation condition for 3 observers (Experiment 4 ). In each case, results are given for optimal and long ISI and for left $(L)$ and right $(R)$ adaptation direction. The stippled line is for the results of the control adaptation condition. Each column is based on 40 observations.
Even though the distance between the two inducing grating stimuli was about $4^{\circ}$, this might not have been large enough. It has been shown that the maximal distance for the operation of the SR mechanism is not fixed, but depends on the spatial frequency and the eccentricity of the inducing stimuli (Baker \& Braddick, 1985; Chang \& Julesz, 1983; Nakayama \& Silverman, 1984). Taking all these variables into account, the separation in the present experiments was still appreciably larger than the maximum separations $(d \max )$ allowed for the SR mechanism. For a stimulus at eccentricities between $2^{\circ}$ and $7^{\circ}$ and with low spatial frequency, a $d$ max value of not more than $1^{\circ}$ would be possible, and more than $4^{\circ}$ was used here.

The stimulus display for the present experiments was set up in such a way that the grating within the two outside windows was seen to move, rather than the windows themselves. The thin vertical strips on each side of the central window gave the impression that the windows remained stationary, while the grating seemed to move from one window to the other across the space of the central window. Thus, the relevant spatial frequency for the above comparisons is the one of the grating.

The conclusion that the LR mechanism was utilized in the present experiments is also supported by the outcome of the fourth experiment. Since the MAE occurred under dichoptic presentation, the LR mechanism must have been involved, if our current understanding of these mechanisms is correct (Anstis, 1980). The MAE reported, but not measured, by Anstis and Moulden (1970) may also have involved the LR mechanism, since it was also present with dichoptic stimulation. The present task did not require an estimate of the strength of the induced MAE, nor was the potentially short duration $(2-3 \mathrm{sec})$ detrimental to the given task, since the judgments had to be made after less than $1 \mathrm{sec}$.

The existence of a MAE for the LR motion mechanism bears on the old question of the similarity of the existing motion mechanisms. Kolers $(1963,1972)$ concluded that the mechanisms for real motion and apparent motion could not be the same, since they have different characteristics. In this case, a really moving object could mask an object in its path, although an apparently moving one could not. But, supported by the facts that they are phenomenally very similar or sometimes identical, and that many neurons respond similarly to real and apparent motion (see, e.g., Barlow \& Levick, 1965; Vautin \& Berkley, 1977), other researchers have maintained that the two are basically the same (Anstis \& Moulden, 1970; Clatworthy \& Frisby, 1973; Frisby, 1972). More recently, it was shown that real (SR) and apparent (LR) motion mechanisms interact and cancel each other out (Green, 1983; Green \& von Grünau, 1983; Gregory \& Harris, 1984). Thus, they must have in common some very important aspects of their organization. The present paper demonstrates that they resemble each other also with respect to their directional aftereffects, thus removing one of the distinguishing criteria. The two mechanisms, therefore, seem in many ways to have rather similar characteristics, but to be 
responsible for different spatial and temporal ranges. Possibly, the LR mechanism includes, in addition, more central systems that are essentially binocular and integrate over larger spatial extents. This integration includes direction selectivity with its adaptability. It is perhaps too simple to attribute only SR motion to the operation of "hardwired" motion detectors, while leaving LR motion to some undefined "higher order" central processes. It is likely that LR motion is also handled by motion detectors which are "hard-wired" but more centrally located.

\section{REFERENCES}

Anstis, S. M. (1980). The perception of apparent motion. Philosophical Transactions of the Royal Society, London, B, 290, 153-168.

ANSTIS, S. M., \& MATHER, G. (1985). Effects of luminance and contrast on the direction of ambiguous apparent motion. Perception, 14. 167-179.

Anstis, S. M., \& Moulden, B. P. (1970). Aftereffect of seen movement: Evidence for peripheral and central components. Quarterly Journal of Experimental Psychology, 22, 222-229.

BAKER, C. L., JR., \& BRADDICK, O. J. (1985). Eccentricity-dependent scaling of the limits for short-range apparent motion perception. $\mathrm{Vi}$ sion Research, 25, 803-812.

Banks, W. P., \& Kane, D. A. (1972). Discontinuity of seen motion reduces the visual motion aftereffect. Perception \& Psychophysics, 12, 69-72.

Barlow, H. B., Levick, W. R. (1965). The mechanism of directionally selective units in rabbit's retina. Joumal of Physiology (London), 178, 477-504.

BeCK, J., \& STEvens, A. (1972). An aftereffect of discrete stimuli producing apparent movement and succession. Perception \& Psychophysics, 12, 482-486.

BenNeTt, R. G., \& Westheimer, G. (1985). A shift in the perceived simultaneity of adjacent visual stimuli following adaptation to stroboscopic motion along the same axis. Vision Research, 25, 565-569.

BRADDICK, O. J. (1974). A short-range process in apparent movement. Vision Research, 14, 519-527.
Chang, J. J., \& Julesz, B. (1983). Displacement limits for spatial frequency filtered random-dot cinematograms in apparent motion. $\mathrm{Vi}$ sion Research, 23, 1379-1385.

Clatworthy, J. L., \& Frisby, J. P. (1973). Real and apparent visual movement: Evidence for a unitary mechanism. Perception, 2, 161-164.

FrusBY, J. P. (1972). Real and apparent movement--same or different mechanisms? Vision Research, 12, 1051-1055.

GREEN, M. (1983). Inhibition and facilitation of apparent motion by real motion. Vision Research, 23, 861-865.

GreEN, M., \& VON GrünaU, M. W. (1983). Real and apparent motion: One mechanism or two? Siggraph/Sigart Interdisciplinary Workshop on Motion (Proceedings; pp. 17-22). Baltimore: ACM Siggraph/Sigart.

GREGORY, R. L., \& HARRIS, J. P. (1984). Real and apparent movement nulled. Nature, 307, 729-730.

KoLERs, P. A. (1963). Some differences between real and apparent visual movement. Vision Research, 3, 191-206.

Kolers, P. A. (1972). A problem for theory. Vision Research, 12, 1057-1058.

Mather, G., Cavanagh, P., \& ANSTis, S. M. (1985). A moving display which opposes short-range and long-range signals. Perception, 14, 163-166.

Nakayama, K., \& Silverman, G. H. (1984). Temporal and spatial properties of the upper displacement limit in random dots. Vision Research, 24, 293-299.

Sekuler, R., \& Pantle, A. (1967). A model for aftereffects of seen movement. Vision Research, 7, 427-439.

Shadlen, M., \& Carney, T. (1985). Cyclopean perception of motion without depth cues. Investigative Ophthalmology \& Visual Science, 26(Suppl.), 55.

VAUTIN, R. G., \&ERKLEY, M. A. (1977). Responses of single cells in cat visual cortex to stimulus movement: Neural correlates of visual after-effects. Journal of Neurophysiology, 40, 1051-1065.

WeRTHEIMER, M. (1912). Experimentelle Studien über das Sehen von Bewegung. Zeitschrift fur Psychologie, 61, 161-265.

WoHLgemuth, A. (1911). On the aftereffect of seen movement. British Journal of Psychology (Monograph Supplement), 1, 1-117.

(Manuscript received December 20, 1985; revision accepted for publication May 12, 1986.) 